\title{
Dynamic friction model with thermal and load dependency: modeling, compensation, and external force estimation
}

\author{
Maged Iskandar and Sebastian Wolf
}

\begin{abstract}
A physically-motivated friction model with a parametric description of the nonlinear dependency of the temperature and velocity as well as the dependency on external load is presented. The fully parametric approach extends a static friction model in the gross sliding regime. We show how it can be seamlessly integrated in standard dynamic friction models such as Lund Grenoble (LuGre) and Generalized-Maxwell-Slip (GMS). Parameters of a Harmonic Drive CSD 25 gear are experimentally identified and the final model is evaluated on a dedicated test-bed. We show the integration and effectiveness in dynamic simulation, friction compensation, and external torque estimation.
\end{abstract}

\section{INTRODUCTION}

Actuation performance in robots with gears is always affected by frictional effects. In robotic drivetrains friction is omnipresent and by nature hinders motion, which is in many cases a restrictive effect. A precise knowledge of the friction in the system helps to improve the mechatronic design, increase precision, and gain a more accurate simulation. Recently this topic has got increasing attention in research for industrial robots [1]-[3] , but also for lightweight service robots knowledge of friction is advantageous, especially when physical human robot interaction plays a major role.

Our primary interest is on lightweight service robots where during typical task execution very different actuator states are present. We want to model the friction of the robot DLR David [4], especially in the main actuators FSJ [5] that have Harmonic Drive gears (HD) as the main source of friction [6]. Typical motions consist of many reversals as well as high and low velocities. Also varying loads affect the system due to gravitation, different payloads, and accelerations. Furthermore, the varying motions directly affect the actuator temperature by the heat of the frictional losses, which consequently has a significant variance.

The aforementioned demands are not fully covered in the well known static or dynamic friction models [7]-[11]. Current research addresses the importance and the inclusion of temperature, velocity, and load in static friction models [1], [3], [12]-[15]. Since we have trajectories with many reversals and low velocity portions we aim on a dynamic friction model.

The proposed method in this paper is an extension to a static friction model that can be integrated in common dynamic friction models. The model includes nonlinear viscous, nonlinear thermal, and load dependency. In contrast to the previous approach presented in [16], it is a fully parametric model and includes load dependency. Our proposed method is fully based on experimental identification and is also

All authors are with the DLR - German Aerospace Center, Robotics and Mechatronics Center, Institute of Robotics and Mechatronics, Wessling, Germany. Email: \{maged.iskandar; sebastian.wolf\}@dlr.de verified on hardware. We show the extended dynamic friction model working in experiments. To our knowledge, there is no parametric approach that is able to combine all the aforementioned properties in one model. Not covered in our model are position dependency of actuators like in [3], HD resonance frequencies [17], and HD ripple [18].

We apply and verify our approach on three major robotic applications where a precise friction model can significantly improve the behavior:

- dynamic simulation

- friction compensation

- external torque estimation

The paper is organized as follows. Section II gives an overview on the underlying standard methods for this work. Section III contains the proposed methods. The testbed setup is described in Section IV and the results are presented in Section $\nabla$ The conclusions can be found in Section VI

\section{BACKGROUND}

\section{A. Static Friction Model}

The typical description of friction torque is a combination of certain aspects representing the basic friction characteristics in the sliding regime, such as static friction, Coulomb friction, viscous friction, and Stribeck effect [19]. Generally, static friction can be modeled as an arbitrary function. A common form of the static friction model is expressed as

$$
\begin{aligned}
\tau_{\mathrm{r}, \mathrm{s}}(\dot{q}) & =g(\dot{q})+s(\dot{q}) \\
g(\dot{q}) & =\operatorname{sign}(\dot{q})\left(F_{\mathrm{c}}+\left(F_{\mathrm{s}}-F_{\mathrm{c}}\right) e^{-\left|\dot{q} / v_{\mathrm{s}}\right|^{\delta_{\mathrm{s}}}}\right) .
\end{aligned}
$$

The function $s(\dot{q})$ expresses the velocity strengthening that is well known as viscous friction, and typically it is linear proportional to the relative velocity $\dot{q}$ as $s(\dot{q})=F_{\mathrm{v}} \dot{q}$, with the constant viscous coefficient $F_{\mathrm{v}}$. The function $g(\dot{q})$ contributes as the velocity weakening of the friction torque. The function $g(\dot{q})$ is alternatively called the Stribeck curve because it captures the Stribeck effect, where $F_{\mathrm{c}}$ is Coulomb friction, $F_{\mathrm{s}}$ is static or stiction friction, $v_{\mathrm{s}}$ is Stribeck velocity, and $\delta_{\mathrm{s}}$ is the exponent parameter of the Stribeck nonlinearity. In the Gaussian parametrization as used in [19] and [20] the exponent parameter is $\delta_{\mathrm{s}}=2$. Parameters of the total friction torque $\tau_{\mathrm{r}, \mathrm{s}}$ can be identified easily using the static map between the friction torque and the relative velocity.

\section{B. Disturbance Observer}

Consider the robot dynamics of the form

$$
\boldsymbol{M}(\boldsymbol{q}) \ddot{\boldsymbol{q}}+\boldsymbol{\eta}(\boldsymbol{q}, \dot{\boldsymbol{q}})+\tau_{\mathrm{ext}}=\boldsymbol{\tau}
$$


with the generalized joint coordinates $\boldsymbol{q}$ and the positive definite inertia matrix $\boldsymbol{M}(\boldsymbol{q})$. The function $\boldsymbol{\eta}(\boldsymbol{q}, \dot{\boldsymbol{q}})$ includes the Coriolis $\boldsymbol{C}(\boldsymbol{q}, \dot{\boldsymbol{q}})$ and the gravity forces $\boldsymbol{\tau}_{g}(\boldsymbol{q})$, as well as the friction torques $\tau_{\mathrm{r}}$. Furthermore, the terms $\tau, \tau_{\text {ext }}$ describe the generalized joint forces and external forces, respectively. The total disturbance imposed on the robot manipulator is expressed as

$$
\begin{aligned}
\boldsymbol{\tau}_{d i s} & =\boldsymbol{\eta}(\boldsymbol{q}, \dot{\boldsymbol{q}})+\boldsymbol{\tau}_{\mathrm{ext}} \\
& =\boldsymbol{\tau}-\boldsymbol{M}(\boldsymbol{q}) \ddot{\boldsymbol{q}}
\end{aligned}
$$

For sake of brevity, the following analysis considers the motion of a single joint with an inertia $J$. Hence, $\eta(q, \dot{q})=$ $\tau_{g}(q)+\tau_{\mathrm{r}}$. However, the same is applied for a multi DoF system in a straight forward manner [21], [22]. The velocity based disturbance observer presented in [23] is based on the transformation of variables, in which the intermediate state is selected as a linear combination of the unknown disturbance $\hat{\tau}_{d i s}$ and the measured velocity $\dot{q}$. Accordingly, the estimated disturbance torque $\hat{\tau}_{d i s}$ can be obtained from the actuator velocity and the commanded torque.

$$
\hat{\tau}_{d i s}=\frac{\beta}{(s+\beta)}(\tau+\beta J \dot{q})-\beta J \dot{q}=Q(s) \tau_{d i s},
$$

where $s$ is the Laplace operator. $\beta$ is the cut-off frequency of the filtered disturbance $\hat{\tau}_{d i s}$ through $Q(s)$, which also determines the bandwidth of the torque sensing, see [21]. This structure of the disturbance observer is widely used in motion control systems. The accuracy of external torque estimation with this observer strongly depends on the knowledge of friction in (3). Thus it can benefit from a precise friction model such as the one presented in this paper.

\section{PROposed METHOdS}

\section{A. Friction Model Refinement}

The proposed friction model refinement addresses the gross sliding regime. The nonlinear viscous friction as well as the nonlinear temperature dependency mainly affect the gross sliding regime. We modify $s(\dot{q})$ and $g(\dot{q})$ of Equation (1) with an according nonlinear term to integrate them into Lund-Grenoble model (LuGre) [9] or GeneralizedMaxwell-Slip model (GMS) [10].

\section{B. Nonlinear Viscous Friction}

An expression that describes the viscous part of a static friction model could be arbitrarily formulated depending on the system behavior, e.g. [19], [24].

We use an exponential shaping factor $\delta_{\mathrm{v}}$ in the nonlinearity of the viscous part of the static model:

$$
s(\dot{q})=F_{\mathrm{v}} \operatorname{sign}(\dot{q})|\dot{q}|^{\delta_{\mathrm{v}}} .
$$

In previous work this expression was found to be well suited to model HD viscous friction [16].

\section{Nonlinear Temperature and Load Dependency}

The friction of the HD is highly dependent on the temperature [25]. In [16] we showed that the temperature dependency could be assigned only to the viscous part and the model still qualitatively represents the behavior. We adapted the most general physically motivated static friction curve in II$\mathrm{A}$ and $\mathrm{III}-\mathrm{B}$ to be continuously valid at different operating temperatures. We experimentally validated that the static model in Equation (1) with nonlinear viscous part (5) can be used to express the friction at a single temperature value.

Now we formulate the model parameters in a generic polynomial form with respect to the temperature and the load. In this way we keep the good representation for the whole velocity range including near zero velocity region (the Stribeck effect) and at the same time map the temperature and load nonlinearities. Adding the temperature and the load dependencies, the friction torque can be expressed as

$$
\tau_{\mathrm{r}, \mathrm{s}}\left(\dot{q}, \xi, \tau_{L}\right)=g\left(\dot{q}, \xi, \tau_{L}\right)+s\left(\dot{q}, \xi, \tau_{L}\right) .
$$

Now $g\left(\dot{q}, \xi, \tau_{L}\right)$ is the temperature and load dependent velocity weakening function (Stribeck curve), $s\left(\dot{q}, \xi, \tau_{L}\right)$ is the temperature and load dependent velocity strengthening function (viscous friction), $\xi$ is the temperature, and $\tau_{L}$ is the load torque. The individual parameters can be formulated as

$$
\begin{aligned}
F_{\mathrm{c}}\left(\xi, \tau_{L}\right) & =\sum_{i=0}^{n_{f_{c}, \xi}} f_{\mathrm{c}_{i}} \xi^{i}+\sum_{j=1}^{n_{f_{c}, \tau_{L}}} f_{\mathrm{c}_{j}} \tau_{L}^{j} \\
F_{\mathrm{v}}\left(\xi, \tau_{L}\right) & =\sum_{i=0}^{n_{f_{v}, \xi}} f_{\mathrm{v}_{i}} \xi^{i}+\sum_{j=1}^{n_{f_{v}, \tau_{L}}} f_{\mathrm{v}_{j}} \tau_{L}^{j} \\
v_{\mathrm{s}}\left(\xi, \tau_{L}\right) & =\sum_{i=0}^{n_{v_{s}, \xi}} v_{\mathrm{s}_{i}} \xi^{i}+\sum_{j=1}^{n_{v_{s}, \tau_{L}}} v_{\mathrm{s}_{j}} \tau_{L}^{j} \\
\delta_{\mathrm{v}}\left(\xi, \tau_{L}\right) & =\sum_{i=0}^{n_{v_{s}, \xi}} \delta_{\mathrm{v}_{i}} \xi^{i}+\sum_{j=1}^{n_{v_{s}, \tau_{L}}} \delta_{\mathrm{v}_{j}} \tau_{L}^{j} .
\end{aligned}
$$

$F_{\mathrm{C}}, F_{\mathrm{v}}, v_{\mathrm{s}}$, and $\delta_{\mathrm{v}}$ are the original four parameters that characterize the static friction behavior at a single temperatureload point (1) in polynomial form, depending on temperature and load. Expressing the dependencies in the individual parameters allows us enough flexibility to map the temperature and load dependencies based on our experimental observations. The order of the polynomial $n$ of each dependency $i, j$ can be chosen in each single parameter according to the system behavior and the required accuracy of the friction estimate. One example is shown in Fig. 3. The approach is to scale the velocity dependent static model depending on temperature and load by simply modifying its parameters with respect to these dependencies in a polynomial fashion.

\section{Extension of dynamic friction models}

Particularly in the instant of velocity reversal, a smooth and better description of the friction than in the static friction model is important. Moreover, the friction phenomenon by nature exhibits a nonlinear continuous behavior during velocity zero crossing, unlike the estimated behavior of the static friction models which is discontinuous at velocity reversal. Following this demand, we extend the generic form of a dynamic friction model by adding the nonlinear viscous behavior, the load and the temperature dependencies. Now the dynamic friction toque $\tau_{\mathrm{r}, \mathrm{d}}$ is a generic nonlinear function 
of internal state $z$ (possibly multi-states), $\dot{q}, \xi$, and $\tau_{\mathrm{L}}$.

$$
\tau_{\mathrm{r}, \mathrm{d}}=f\left(\boldsymbol{z}, \dot{q}, \xi, \tau_{\mathrm{L}}\right)
$$

The generic internal state dynamics could be described as a first order differential with the velocity, temperature, and load dependencies,

$$
\frac{d \boldsymbol{z}}{d t}=G\left(\boldsymbol{z}, \dot{q}, \xi, \tau_{\mathrm{L}}\right) .
$$

This state captures the transient response with respect to the velocity. If the velocity is constant then the function $G\left(\boldsymbol{z}, \dot{q}, \xi, \tau_{\mathrm{L}}\right)=0$ and the function $f\left(\boldsymbol{z}, \dot{q}, \xi, \tau_{\mathrm{L}}\right)$ will converge to the static friction torque $\tau_{\mathrm{r}, \mathrm{s}}\left(\dot{q}, \xi, \tau_{\mathrm{L}}\right)$. As we kept the generic form of the functions $g$ and $s$, it is possible to integrate with different models from the literature. The LuGre and GMS models were selected among the generalized empirical friction models as they are widely used in robotic community and promise accurate results, [26][29]. In addition, both model structures provide the ability to model arbitrary steady state friction behavior in sliding regime. In fact we will use this property to include the load and temperature dependencies. The dynamic friction torque in case of the modified LuGre model can be expressed as

$$
\begin{aligned}
\tau_{\mathrm{r}, \mathrm{d}} & =\sigma_{0} z+\sigma_{1} \dot{z}+s\left(\dot{q}, \xi, \tau_{\mathrm{L}}\right), \\
\frac{d z}{d t} & =\dot{q}-\sigma_{0} \frac{|\dot{q}|}{g\left(\dot{q}, \xi, \tau_{\mathrm{L}}\right)} z,
\end{aligned}
$$

where $z$ is the internal friction state and can be interpreted as the average deflection of the micro bristles, $\sigma_{0}$ is the bristle stiffness, and $\sigma_{1}$ is the micro-damping coefficient.

Similarly, the dynamic friction torque in case of the modified GMS model given as a parallel connection of $N$ single state friction models, then $z_{i}$ is the $i^{t h}$ element of the state vector $z$. The internal states equations are determined by the following. In case of a sticking element, the state equation is given by:

$$
\frac{d z_{i}}{d t}=\dot{q}
$$

and the element remains sticking until $z_{i}=g_{i}\left(\dot{q}, \xi, \tau_{\mathrm{L}}\right)$. In case of a slipping element, the state equation is given by:

$$
\frac{d z_{i}}{d t}=\operatorname{sgn}(\dot{q}) C_{i}\left(1-\frac{z_{i}}{g_{i}\left(\dot{q}, \xi, \tau_{\mathrm{L}}\right)}\right),
$$

where $C_{i}$ is the attraction parameter which is a gain that determines how fast $z_{i}$ converges to $g_{i}\left(\dot{q}, \xi, \tau_{\mathrm{L}}\right)$ and $g_{i}\left(\dot{q}, \xi, \tau_{\mathrm{L}}\right)$ is the velocity weakening (Stribeck) function for element $i$. The element remains slipping until the velocity goes through zero. The friction torque is given as the summation of the outputs of the $N$ elementary state models plus the viscous part as

$$
\tau_{\mathrm{r}, \mathrm{d}}=\sum_{i=1}^{N} k_{i} z_{i}(t)+s\left(\dot{q}, \xi, \tau_{\mathrm{L}}\right) .
$$

The number of unknown parameters in the GMS model is affected by the number of Maxwell elements comprised. Each element is characterized by a stiffness $k_{i}$, an attraction parameter $C_{i}$, and a velocity weakening function $g_{i}\left(\dot{q}, \xi, \tau_{\mathrm{L}}\right)$. However, we reduced the number of the unknown parameters in the implementation by assuming $g_{i}=\mu g$ and $C_{i}=\mu C$, where $\mu$ is a scaling factor. In this paper we will assume an equal scaling among the Maxwell elements, thus $\mu=1 / N$. The identification of the parameters of LuGre and GMS models is carried out in two different regimes, the pre-sliding and the gross sliding phases.

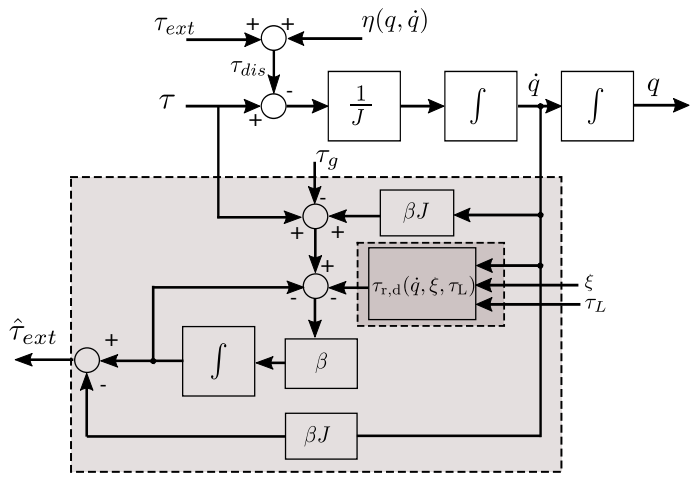

Fig. 1: The implementation of external torque estimation includes the proposed model marked in dark gray.

\section{E. External torque estimation}

From (3) it can be seen that the estimated disturbance by the observer is a result of different components, but the observer is capable to estimate a single output. As we are interested in the external torque $\tau_{\text {ext }}$ and it is hidden inside the total disturbance torque $\tau_{d i s}$, the other nonlinear effects need to be eliminated in order to obtain the external torque as

$$
\tau_{\text {ext }}=\tau_{\text {dis }}-\eta(q, \dot{q}) .
$$

In case of single joint/link $\eta(q, \dot{q})$ equals to gravitational and friction nonlinearities. The unknown external reaction torque can be computed as

$$
\tau_{\text {ext }}=Q(s)\left[\tau+\beta J \dot{q}-\tau_{g}(q)-\tau_{r, d}\left(\dot{q}, \xi, \tau_{L}\right)\right]-\beta J \dot{q} .
$$

For the proposed structure of the external torque estimation, it is necessary to know precisely the gravity and the friction torques. In other words, the precision of the external torque estimation is highly dependent on how accurate the estimation of the friction effect is, as it is an additional source of disturbance and having nonlinear effect. In spite of these, the proposed method can provide a good estimate of the external torque at different operating conditions as it is already considered during the estimation of the friction torque. The proposed integration of the disturbance observer together with the dynamic friction model is depicted in Fig. 1 .

\section{Test SetuP}

The mechanical design of the test-bed setup is depicted in Fig. 22 For a detailed description please refer to [16]. The gear in this setup is a CSD 25 of Harmonic Drive AG with gear ratio 1:80 and rated torque of $75 \mathrm{Nm}$, which is also the main gear of the FSJ. A Lorenz Messtechnik $\mathrm{GmbH}$ DR-2643 torque sensor with range of $\pm 5 \mathrm{Nm}$ is attached to the gear input. At the gear output another DR2643 torque sensor with range of $\pm 200 \mathrm{Nm}$ is connected, which is attached rigidly to an absolute high resolution 


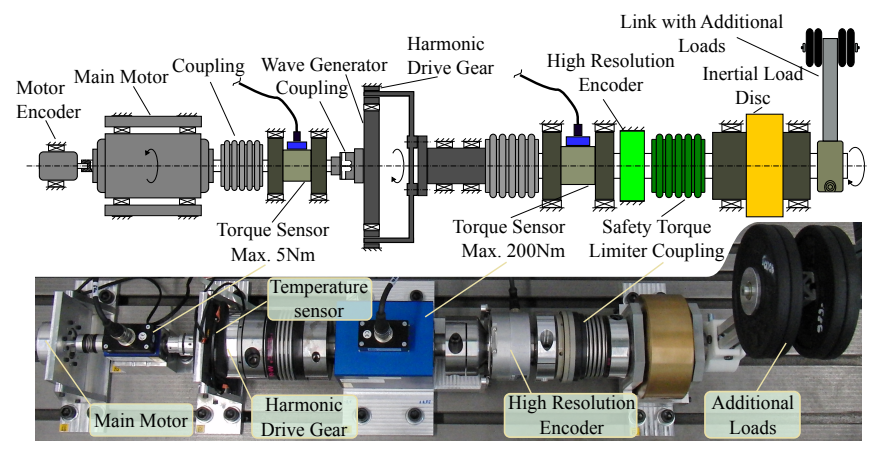

Fig. 2: FSJ principal axes drive train test-bed setup (bottom) and the corresponding schematic diagram (top). The mechanical construction of the test-bed allows to build multi-configuration setups, additional loads can be attached according to the demand.

25 bit Heidenhain encoder. The setup of [16] was altered by the following changes. The motor unit is now a ILM50x8, which is the same as in the FSJ of axis 1 to 4 in the robot David. Furthermore, the setup is augmented by a lever with a weight in order to investigate gravitational load effects of up to $11 \mathrm{Nm}$ on the system. Two temperature sensors of type MCP9808 are placed on the housing surface of the HD in order to acquire the gear temperature.

\section{Results}

\section{A. Nonlinear Temperature and Load Dependent Friction Model}

Intensive experimental measurements have been carried out to get insight and separate the dependencies in the velocity, temperature, and load. The collection of thirty-four different constant velocity points covering the considered velocity range over different constant temperatures allows us to discover the exponential velocity strengthening shape function (5). Moreover, it helps to identify different temperature independent friction curves to have an idea about the parameters change with respect to the temperature Fig. 3 b).

As it was shown in [16], nonlinear viscous friction is present in a Harmonic Drive gear. The measurements were chosen with higher density at low velocities to increase the accuracy of the identified parameters in this region that represent both the nonlinearity of the viscous friction and the Stribeck effect. The temperature deviation during every individual experiment was kept $\leq \pm 0.1^{\circ} \mathrm{C}$, which is assumed as an acceptable uncertainty.

Despite the number of the parameters that have involved in the model, our approach is to separate the different effects and split up the identification problem to relevant parameters. Thus, the identification process of the model parameters has been done in three steps.

- The first step is to identify the velocity and temperature dependencies represented in (6) and (7). The identification problem of the model can be formulated in terms of least squares as

$$
\hat{p}=\min _{p} \sum_{k=1}^{N}\left(\hat{\tau}_{\mathrm{r}, \mathrm{s}}(\dot{q}, \xi, p)-\tau_{\mathrm{r}, \mathrm{s}}(\dot{q}, \xi)\right)^{2},
$$

TABLE I: Identified parameters in case of second-order temperature dependent static curve.

\begin{tabular}{lrrr}
\hline$F_{\mathrm{s}}$ & $f_{\mathrm{s} 0}$ & & \\
\hline$F_{\mathrm{c}}\left[10^{-2}\right]$ & 4.48 & & \\
\hline \multirow{2}{*}{$f_{\mathrm{v} 0}\left[10^{-1}\right]$} & $f_{\mathrm{c} 1}$ & $f_{\mathrm{c} 2}$ \\
& -35.83 & 10.28 & -0.06 \\
\hline$v_{\mathrm{s} 0}\left[10^{-1}\right]$ & 29.58 & $f_{\mathrm{v} 1}$ & $f_{\mathrm{v} 2}$ \\
& $v_{\mathrm{s} 0}$ & $v_{\mathrm{s} 1}$ & 0.01 \\
\hline$\delta_{\mathrm{v}}\left[10^{-2}\right]$ & -53.10 & 5.49 & -0.07 \\
& $\delta_{\mathrm{v} 0}$ & $\delta_{\mathrm{v} 1}$ & $\delta_{\mathrm{v} 2}$ \\
& 90.49 & -3.77 & 0.07 \\
\hline
\end{tabular}

where the parameter vector $p \in \mathbb{R}^{n_{\mathrm{r}}}$ depends on the order of the assumed polynomials.

- The second step is to fix the velocity-temperature model parameters and to add the load dependent parameters fitting the second part of the parameters expressions (7). The trust-region reflective algorithm that is available in optimization toolbox of Matlab is employed in the identification process of the first and the second step that represent the static friction model (6). The identified parameters are summarized in Table I.

- In the third step the dynamical parameters of the LuGre and GMS models are identified by executing low velocity experiments that include mainly pre-sliding phases similar to the one shown in Fig. 5. The pattern search algorithm has been used to identify the presliding parameters of the modified LuGre model (10) and the modified GMS model (13).

Fig. 3 b) represents the behavior of the individual parameters with respect to the temperature. The markers indicate the parameters of the temperature independent models that have been identified at single temperature values. A second order polynomial is found to be sufficient to represent the parameter variation in the temperature (depicted in solid lines). The load dependency is assumed to be linear for simplicity since it shows acceptable results as in Fig. 4 . However, this could be improved by assuming a higher order load dependency. The overall output of the proposed model is illustrated in Fig. 3. (c) and Fig. 4 for the temperature and load dependencies, respectively.

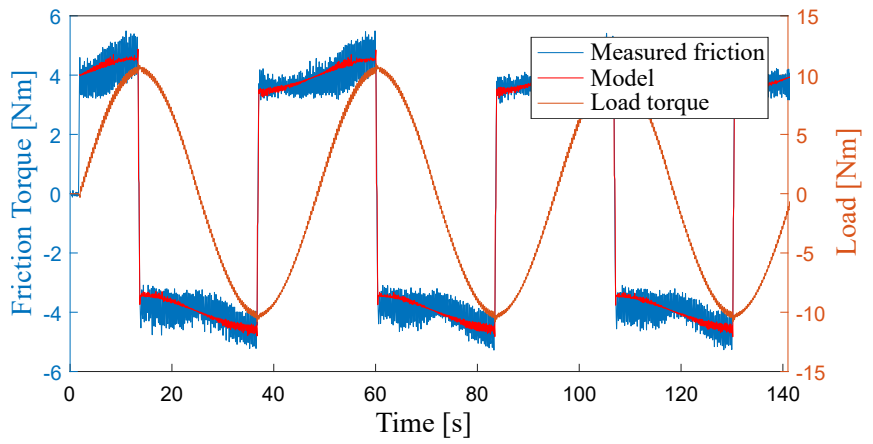

Fig. 4: Load dependency experiment at constant velocity.

\section{B. Dynamic Simulation}

The identified parameters of the two dynamic friction models together with the torque constant and the motor 

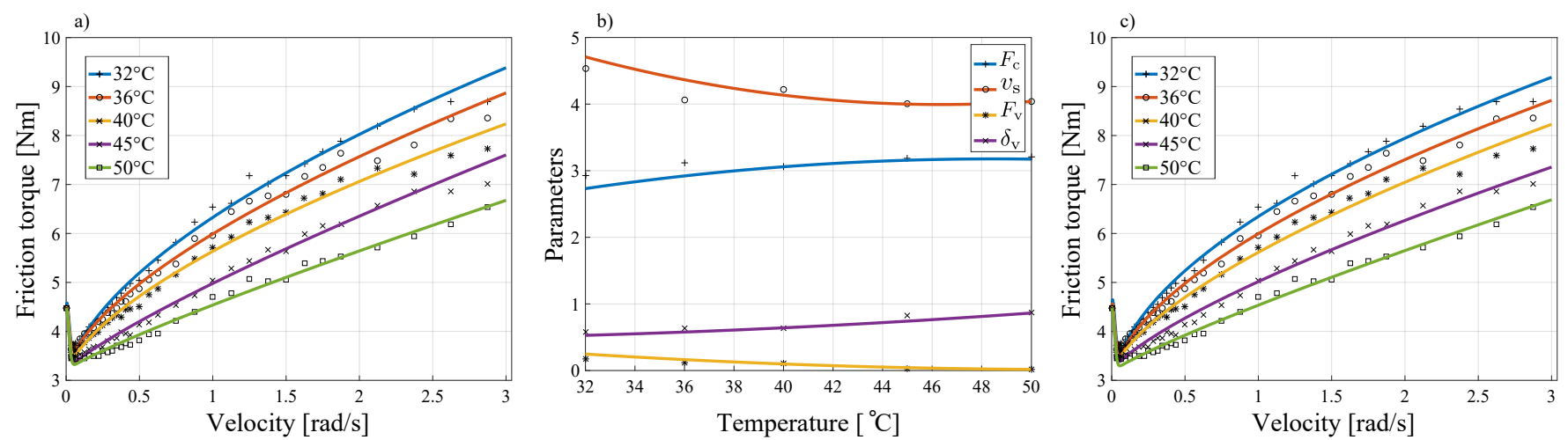

Fig. 3: The identified static friction models at different individual temperature values with the experimental data are shown in (a). While (b) depicts the model parameter change with respect to the temperature indicated with markers and in solid lines the behavior of each parameter using a second order polynomial to map the temperature dependency as in Equation 77. The output of the temperature dependent static model with the experimental data is shown in (c).

inertia are used to build a dynamical model of the test setup. The dynamical simulation capabilities are validated for the modified LuGre and modified GMS with respect to the experimental measurements. Two chirp signals having the same increasing frequency, but with different constant amplitudes, are used as the commanded torque. This results in one trajectory with low velocity Fig. 5(a-c) and one with high velocity Fig. 5(d-f). The same signals are used as input signals for the model. At the same time the measured temperature and load signals were fed to the model in order to have similar simulation conditions. The comparison between the commanded and the applied torque is shown in Fig. 5 (a,d), where the applied torque is calculated as the product of measured current and motor constant.

Fig. 5 $(b, e)$ shows the open loop velocity responses of the models and the measured data. It can be clearly seen that the commanded motor torque is continuous, while the resulting velocity response is not due to the stiction phases. The proposed models show high accuracy estimating the actual velocity response including the discontinuity behavior.

\section{Friction Compensation}

Model based friction compensation benefits from accurate friction estimation ${ }^{1}$ The model (8) has been realized to be compatible with a real-time system. Assuming all the parameters of the system are known and neglecting the friction torque in (2), we can design a simple PD controller with additional feed-forward terms to compensate the inertial and gravity torques as

$$
\tau=J \ddot{q}_{\mathrm{d}}+k_{\mathrm{p}} \tilde{q}+k_{\mathrm{d}} \dot{\tilde{q}}+\tau_{g}(q)+\psi \tau_{\mathrm{r}, \mathrm{d}}\left(\boldsymbol{z}, \dot{q}, \xi, \tau_{\mathrm{L}}\right),
$$

where $\tilde{q}=q_{\mathrm{d}}-q$ and $\dot{\tilde{q}}=\dot{q}_{\mathrm{d}}-\dot{q}$ are the position and velocity errors, respectively, and $\psi$ is a logic variable 0 or 1 to enable the friction compensation. The desired position $q_{d}$ is assumed to be smooth and with bounded derivatives up to the second order.

Our goal is to validate the friction compensation capability. Thus the controller (17) is implemented in real time with sampling rate $3 \mathrm{kHz}$ and the gains $k_{\mathrm{p}}$ and $k_{\mathrm{d}}$ are calculated to respect the transient time requirements of rise time $0.04 \mathrm{~s}$

\footnotetext{
${ }^{1}$ see also application video https://youtu.be/TtGtr9OE9tk
}

and a maximum overshoot of $6 \%$ for the step response. Fig. 6 shows the results of the friction compensation at low and high velocity in comparison to the uncompensated scenario. The same controller parameters are used in both uncompensated experiments and while the friction compensation is active. The proposed dynamic-model based friction compensation is examined with two different sinusoidal trajectories $q_{d}(t)=a_{q} \sin \left(\omega_{q} t\right)$ with $a_{q}=\{0.4 ; 2.4\}$ and $\omega_{q}=1.5$ at three different temperature values. Results of two temperature values are depicted in Fig. 6

A significant reduction of the tracking error is observed while activating the friction compensator. The maximum tracking error of the proposed models with respect to the desired value is shown in Fig.77 It can be seen that neglecting the friction can lead to an position error of $29 \%$ to $35 \%$ from the commanded position in low velocity case, depending on the temperature. The remaining position error could be a result of the parameter uncertainty and measurement noise as well as additional unmodeled dynamics and effects. Moreover, we observe that the position error of Lugre and GMS is not significantly different while compensating friction. Note that, if the back-drivability is desired, GMS based model has a smoother zero crossing behavior.
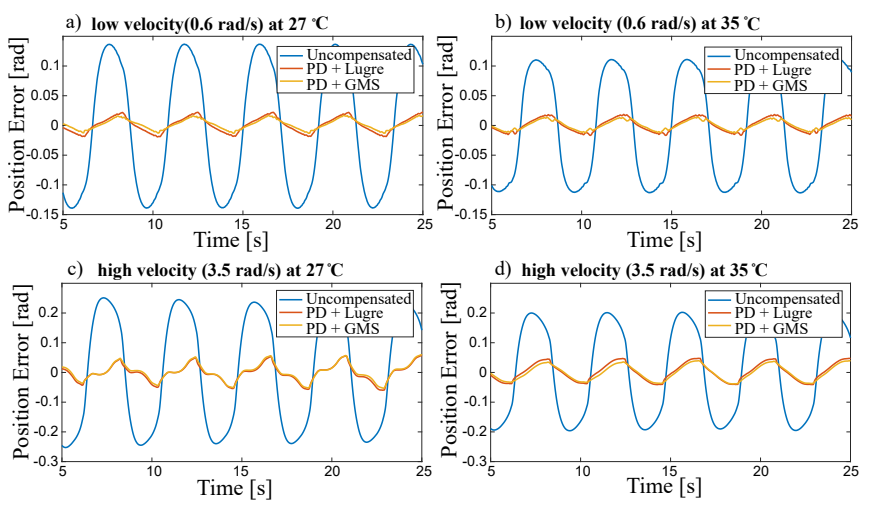

Fig. 6: The experimental results of the friction compensation using modified LuGre and GMS models. The position errors at low velocity (peak $0.6 \mathrm{rad} / \mathrm{s}$ ) and two different operating temperatures $27^{\circ} \mathrm{C}$ and $35^{\circ} \mathrm{C}$ are shown in (a) and (b), respectively. Similar results for the high velocity case (peak $3.5 \mathrm{rad} / \mathrm{s}$ ) are shown in (c) and (d). 
a)
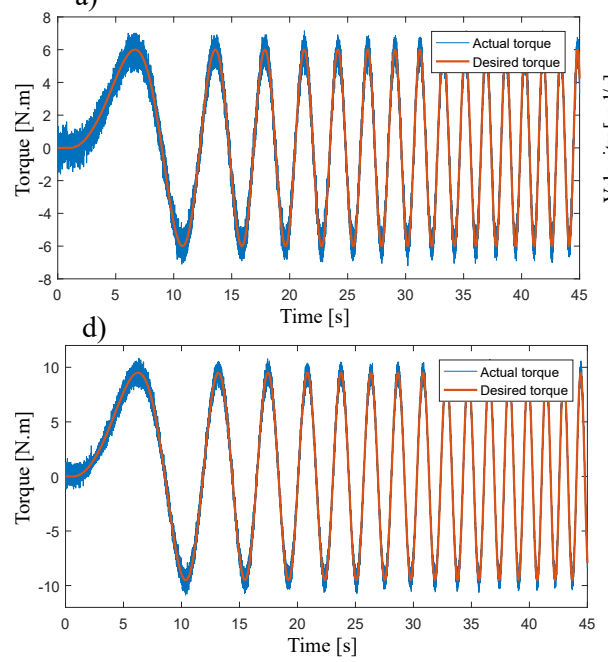

b)
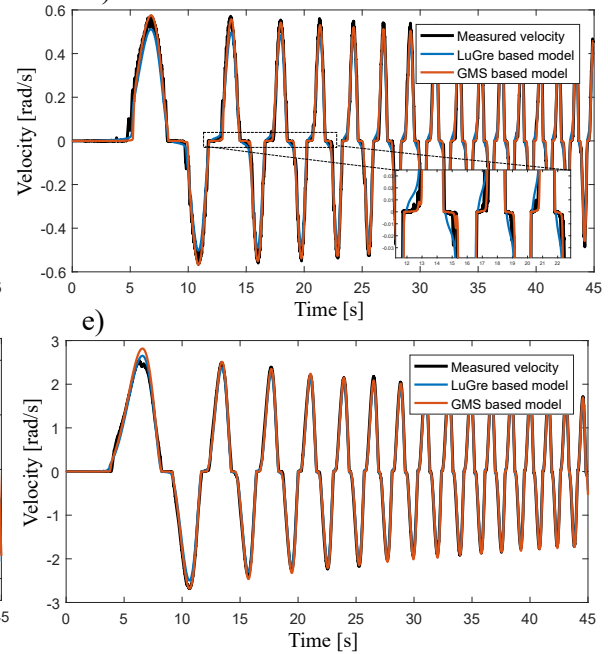

c)
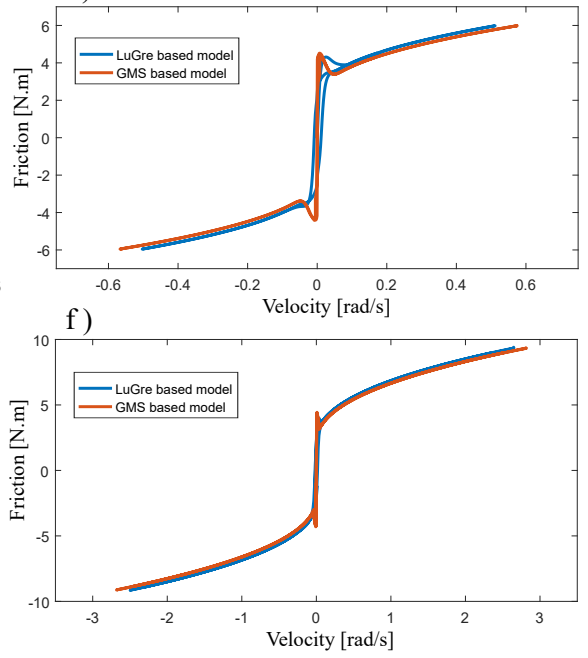

Fig. 5: The evaluation experiment of drive-train using chirp excitation signal with low (a,b,c) and high (d,e,f) amplitude resulting in low and high velocity. The commanded and actual torques are shown in (a,d). The measured and modeled velocities as a result of dynamic friction model (LuGre) with modified nonlinear viscous behavior in comparison with GMS $(\mathrm{b}, \mathrm{e})$. The corresponding estimated friction torques are shown in $(\mathrm{c}, \mathrm{f})$.

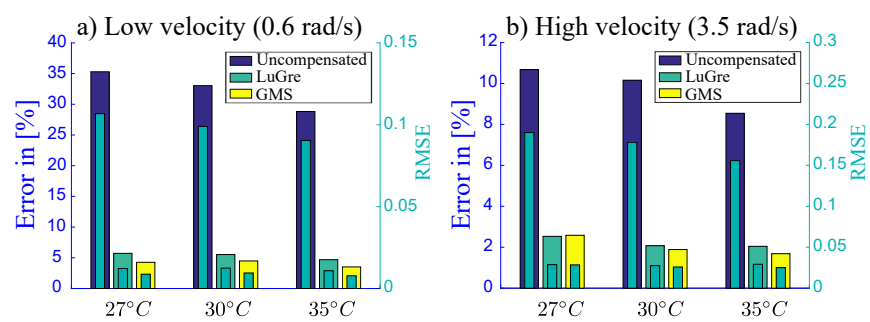

Fig. 7: The maximum position error as a percentage from the desired position at different operating temperatures are shown, the thin bars show the corresponding RMSE of the trajectory.

\section{External torque estimation}

The integration of the disturbance observer is evaluated with variable velocity and load. The gravitational torque is excluded from (15) and it is assumed to be an unknown external variable load. The link side torque sensor is used to compare the estimated external torque $\hat{\tau}_{\text {ext }}$ with the applied external torque.

From Fig. 8 it is obvious that including the friction model plays crucial role in estimating external torque. The estimated external toque error is less than $1 \mathrm{Nm}$, which allows the implementation of an impedance control or to use the estimated external torque in more sophisticated algorithms without the need of an extra torque sensor.

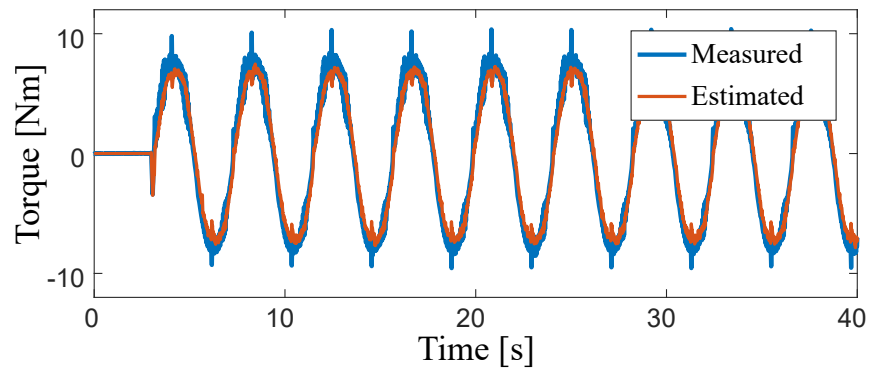

Fig. 8: External torque estimation.

\section{CONCLUSIONS}

A physically-motivated friction model with parametric description of the nonlinearity of the temperature dependency, velocity, and on the dependency on external load is presented. It is shown how this model can be integrated in the dynamic models of LuGre and GMS. The parameter-identification and evaluation were executed on a dedicated testbed. The model with LuGre and GMS was evaluated in three robotic applications. First it was shown that in a dynamic simulation a very good correspondence also in the zero crossing behavior was achieved. Second the maximum position error in sinusoidal movements with friction compensation dropped from $35 \%$ in the uncompensated case to $6 \%$ and $4 \%$ with the LuGre and GMS model, respectively. As a third application the external torque estimation was investigated with a newly proposed integration in the observer structure and showed also a good accuracy with approximately $14 \%$ error.

Future work will be on the extension of the experimental evaluation to a wider range of temperature, velocity, and load. Furthermore, additional hardware units will be examined with a focus on the variance. Finally, the extended dynamic friction model will be implemented on the robot David to improve friction compensation and external torque estimation.

\section{REFERENCES}

[1] J.-C. Mar, "Friction modelling and simulation at system level: Considerations to load and temperature effects," Proceedings of the Institution of Mechanical Engineers, Part I: Journal of Systems and Control Engineering, vol. 229, no. 1, pp. 27-48, 2015.

[2] L. Simoni, M. Beschi, G. Legnani, and A. Visioli, "Friction modeling with temperature effects for industrial robot manipulators," in 2015 IEEE/RSJ International Conference on Intelligent Robots and Systems (IROS). IEEE, 2015, pp. 3524-3529.

[3] A. Wahrburg, S. Klose, D. Clever, T. Groth, S. Moberg, J. Styrud, and H. Ding, "Modeling speed-, load-, and position-dependent friction effects in strain wave gears," in Robotics and Automation (ICRA), 2018 IEEE International Conference on, 2018, pp. 2095-2102. 
[4] M. Grebenstein, A. Albu-Schäffer, T. Bahls, M. Chalon, O. Eiberger, W. Friedl, R. Gruber, U. Hagn, R. Haslinger, H. Höppner, S. Jörg, M. Nickl, A. Nothhelfer, F. Petit, B. Pleintinger, J. Reil, N. Seitz, T. Wimböck, S. Wolf, T. Wüsthoff, and G. Hirzinger, "The DLR Hand Arm System," in Robotics and Automation (ICRA), 2011 IEEE International Conference on, 2011, pp. 3175-3182.

[5] S. Wolf, O. Eiberger, and G. Hirzinger, "The DLR FSJ: Energy based design of variable stiffness joints," in Robotics and Automation (ICRA), 2011 IEEE International Conference on. Shanghai, China: IEEE, May 2011, pp. 5082-5089.

[6] S. Wolf and J.-E. Feenders, "Modeling and benchmarking energy efficiency of Variable Stiffness Actuators on the example of the DLR FSJ," in Intelligent Robots and Systems (IROS), 2016 IEEE/RSJ International Conference on, 2016, pp. 529-536.

[7] B. Armstrong-Hélouvry, Control of Machines with Friction, T. Kanade, Ed. Kluwer Academic Publishers, 1991

[8] P. R. Dahl, "A solid friction model," DTIC Document, Tech. Rep., 1968.

[9] C. C. de Wit, H. Olsson, K. J. Åström, and P. Lischinsky, "A new model for control of systems with friction," Automatic Control, IEEE Transactions on, vol. 40, no. 3, pp. 419-425, Mar 1995.

[10] V. Lampaert, J. Swevers, and F. Al-Bender, "Modification of the Leuven integrated friction model structure," IEEE Transactions on Automatic Control, vol. 47, no. 4, pp. 683-687, Apr 2002.

[11] F. Al-Bender and J. Swevers, "Characterization of friction force dynamics," IEEE Control Systems, vol. 28, no. 6, pp. 64-81, Dec 2008.

[12] A. C. Bittencourt, E. Wernholt, S. Sander-Tavallaey, and T. Brogårdh, "An extended friction model to capture load and temperature effects in robot joints," in Intelligent Robots and Systems (IROS), 2010 IEEE/RSJ International Conference on. IEEE, Oct 2010, pp. 6161-6167.

[13] P. Hamon, M. Gautier, and P. Garrec, "New dry friction model with load- and velocity-dependence and dynamic identification of multiDOF robots," in 2011 IEEE International Conference on Robotics and Automation, May 2011, pp. 1077-1084.

[14] T. Verstraten, G. Mathijssen, R. Furnémont, B. Vanderborght, and D. Lefeber, "Modeling and design of geared dc motors for energy efficiency: Comparison between theory and experiments," Mechatronics, vol. 30, no. Supplement C, pp. 198-213, 2015.

[15] L. Simoni, M. Beschi, G. Legnani, and A. Visioli, "On the inclusion of temperature in the friction model of industrial robots," IFACPapersOnLine, vol. 50, no. 1, pp. 3482-3487, 2017, 20th IFAC World Congress.
[16] S. Wolf and M. Iskandar, "Extending a dynamic friction model with nonlinear viscous and thermal dependency for a motor and Harmonic Drive gear," in Robotics and Automation (ICRA), 2018 IEEE International Conference on, 2018, pp. 783-790.

[17] T. D. Tuttle and W. P. Seering, "A nonlinear model of a harmonic drive gear transmission," IEEE Transactions on Robotics and Automation, vol. 12 , no. 3, pp. 368-374, 1996.

[18] F. H. Ghorbel, P. S. Gandhi, and F. Alpeter, "On the kinematic error in harmonic drive gears," Journal of Mechanical Design, vol. 123, no. 1, pp. 90-97, March 2001.

[19] B. Armstrong-Helouvry, Control of machines with friction. Springer Science \& Business Media, 2012, vol. 128.

[20] A. Tustin, "The effects of backlash and of speed-dependent friction on the stability of closed-cycle control systems," Electrical Engineers - Part IIA: Automatic Regulators and Servo Mechanisms, Journal of the Institution of, vol. 94, no. 1, pp. 143-151, May 1947.

[21] T. Murakami, F. Yu, and K. Ohnishi, "Torque sensorless control in multidegree-of-freedom manipulator," IEEE Transactions on Industrial Electronics, vol. 40, no. 2, pp. 259-265, April 1993.

[22] I. S. Khalil, E. D. Kunt, and A Sabanovic, "Sensorless torque estimation in multidegree-of-freedom flexible systems," in 2009 35th Annual Conference of IEEE Industrial Electronics. IEEE, 2009, pp. 2354-2359.

[23] K. Ohnishi, M. Shibata, and T. Murakami, "Motion control for advanced mechatronics," IEEE/ASME transactions on mechatronics, vol. 1, no. 1, pp. 56-67, 1996.

[24] B. Bona and M. Indri, "Friction compensation in robotics: an overview," in Decision and Control, 2005 and 2005 European Control Conference. CDC-ECC'05. 44th IEEE Conference on. IEEE, 2005 pp. 4360-4367.

[25] Harmonic Drive AG, Engineering Data Catalogue, 2017.

[26] K. Johanastrom and C. Canudas-De-Wit, "Revisiting the lugre friction model," IEEE control Systems, vol. 28, no. 6, pp. 101-114, 2008.

[27] V. Lampaert, F. Al-Bender, and J. Swevers, "A generalized maxwellslip friction model appropriate for control purposes," in Physics and Control, 2003. Proceedings. 2003 International Conference, vol. 4 IEEE, 2003, pp. 1170-1177.

[28] T. Tjahjowidodo, F. Al-Bender, and H. Van Brussel, "Friction identification and compensation in a dc motor," in submitted to 16th IFAC World Congress, Prague, 2005.

[29] L. Freidovich, A. Robertsson, A. Shiriaev, and R. Johansson, "Lugremodel-based friction compensation," IEEE Transactions on Control Systems Technology, vol. 18, no. 1, pp. 194-200, 2010. 